\title{
El ciclo Zilch en el contexto de la termodinámica de tiempos finitos: eficiencia y potencia de salida
}

\author{
Delfino Ladino-Luna1* ${ }^{*}$ Juan Carlos Chimal-Eguía² y Ricardo T. Páez-Hernández1 \\ ${ }^{1}$ Universidad Autónoma Metropolitana-A, Física de Procesos Irreversibles, Ciencias Básicas, Av. San Pablo 180, Col. \\ Reynosa, 02200, Azcapotzalco, Ciudad de México, México (correo-e: dll@azc.uam.mx, phrt@azc.uam.mx). \\ ${ }^{2}$ Centro de Investigación en Computación del IPN, Av. Juan de Dios Batiz s/n esq Miguel Othón de Mendizabal, Col. San \\ Pedro Zacatenco, C. P. 07738, Ciudad de México, México (correo-e: jchimale@gmail.com).
}

* Autor a quien debe ser dirigida la correspondencia.

Recibido Jul. 13, 2020; Aceptado Sep. 14, 2020; Versión final Nov. 13, 2020, Publicado Abr. 2021

\begin{abstract}
Resumen
El principal objetivo del presente estudio es examinar el ciclo Zilch desde el punto de vista de la termodinámica de tiempos finitos. Se propone una modificación del ciclo original, incluyendo explícitamente la diferencia entre temperaturas de trabajo y temperaturas de las fuentes de calor. Se construyen las expresiones de potencia de salida y eficiencia del ciclo con ciertas variables neutras, definidas como razones entre temperaturas de trabajo y de las fuentes. Se establecen restricciones para simplificar las expresiones obtenidas de potencia y eficiencia, y se imponen restricciones apropiadas para hallar sus regiones de existencia física. Los resultados muestran la viabilidad de los procedimientos usados, y la posibilidad que el ciclo estudiado sirva como modelo teórico para representar una planta con regeneración de calor. Se concluye que es posible describir el comportamiento de una máquina o planta de poder a través de la descripción del comportamiento de la potencia y la eficiencia.
\end{abstract}

Palabras clave: eficiencia; endoreversibilidad; optimización; potencia de salida; ciclo Zilch

\section{The Zilch cycle in the context of finite-time thermodynamics: efficiency and power output}

\begin{abstract}
The primary objective of the present study is to examine the Zilch cycle from a finite-time thermodynamics perspective. A modification of the original cycle is proposed by explicitly including the difference between working temperatures and heat source temperatures. Expressions of power output and cycle efficiency are constructed with neutral variables defined as ratios between working and temperature sources. To simplify the obtained expressions for power and efficiency, appropriate restrictions are imposed to find physical existence regions. The results show the viability of the procedures used and show that the cycle studied can serves as a theoretical model for heat regeneration plants. It is concluded that it is possible to successfully describe the behavior of regeneration plants by describing power and efficiency dynamics.
\end{abstract}




\section{INTRODUCCIÓN}

Como se sabe, un proceso cuasiestático es la idealización de un proceso real, de manera que el sistema que se este estudiando se encuentre muy cerca de un estado de equilibrio. Pero un proceso tal necesita un tiempo muy grande para realizarse. Un proceso reversible es necesariamente cuasiestático, pero un proceso cuasiestático no necesariamente es reversible. En la termodinámica clásica de equilibrio generalmente los procesos que se estudian se consideran reversibles, y los procesos de transferencia de calor entre el sistema y sus fuentes se consideran instantáneos. Pero, en los procesos reales las condiciones de reversibilidad son prácticamente nulas y los procesos de transferencia de calor ocurren en un tiempo medible. Actualmente se estudian teóricamente diferentes sistemas termodinámicos en el marco de la termodinámica de tiempos finitos, que se ha desarrollado a partir del trabajo pionero de Curzon y Ahlborn (1975) en el que se analizó un ciclo de Carnot modificado (conocido ahora como ciclo de Curzon-Ahlborn), tomando en cuenta el tiempo necesario para la transferencia de calor; y que tiene como antecedente los trabajos de Chambadal (1957) y Novikov (1958). Recientemente ha sido obtenida la eficiencia del ciclo de Carnot modificado, ciclo de CurzonAhlborn, como un caso particular de una expresión más general (Ponmurugan, 2019).

Como se puede observar en la literatura, se han realizado diversos estudios sobre el desempeño de dispositivos convertidores de energía a partir de la hipótesis de transferencia calor en un tiempo finito. Particularmente se han estudiado diversos criterios de desempeño del ciclo de Curzon-Ahlborn (Gutkowicz et al., 1978; Rubin, 1980; Angulo-Brown, 1991); se ha aplicado a ciclos que modelan máquinas reales (Mozurkevich, 1982; Angulo-Brown et al., 1994; Lefft, 1987); se ha usado en el análisis de sistemas en estado estable (Santillán et al., 2001; Ladino-Luna et al., 2013; Páez-Hernández et al., 2018); y se han propuesto simplificaciones de los parámetros variables del ciclo de Curzon-Ahlborn (Agrawal, 2009; Páez-Hernández et al., 2016); entre otros muchos tópicos y autores. Además, con esa misma hipótesis (transferencia calor en un tiempo finito) se ha abordado recientemente un estudio de ciclos para máquinas térmicas como un modelo realista de la máquina Stirling (Lanzetta et al., 2016), una nueva optimización del ciclo Diesel (Zhu et al., 2019), un estudio de plantas hibridas solares (Merchán et al., 2020), entre muchos otros tópicos. La literatura sobre diversos estudios de ciclos térmicos en el contexto de la termodinámica de tiempos finitos es abundante, de manera que las referencias comentadas son solo una pequeña muestra.

Es importante mencionar que en los trabajos antes citados, aún los más recientes, se busca generalmente una función objetivo, y la eficiencia obtenida a partir de ella, como función de algún parámetro controlable, con el objeto de analizar diversos aspectos del funcionamiento de una máquina térmica, pero en pocos de se hace un análisis de las regiones de existencia de las funciones objetivo. Además, se parte de la idea de analizar un ciclo que modele alguna máquina térmica o sistema convertidor de energía. El problema inverso, analizar un ciclo teórico susceptible de usarse para representar algún sistema termodinámico no conocido, no es abordado. Sin embargo podría intentarse un estudio en esa dirección, de manera semejante al estudio de diversos ciclos conocidos que, como se sabe, son idealizaciones de los ciclos reales que representan la manera como trabajan diversos sistemas termodinámicos convertidores de energía.

Debe también tomarse en cuenta que los ciclos termodinámicos estudiados comúnmente se componen de un pequeño número de transformaciones isotérmicas, adiabáticas, de presión y volumen constante, suponiendo como fluido de trabajo generalmente un gas ideal. Los más estudiados son los ciclos de Carnot modificado (ciclo de Curzon-Ahlborn), Stirling, Otto, Diesel y Joule-Brayton; incluyendo ciclos de vapor y refrigeradores para fluidos de trabajo reales. Cualquier ciclo adicional compuesto por las mismas transformaciones simples, y cualquier análisis extendido de ciclos conocidos, deberían ser adiciones bienvenidas al repertorio, ya que brindan más oportunidades para la discusión sobre el comportamiento y posibilidades de diseño de máquinas y sistemas convertidores de energía.

Por otro lado, con el objeto de mejorar el rendimiento de las plantas de potencia, se ha buscado la forma de utilizar el calor residual para mejorar el aprovechamiento de la energía suministrada en diversos procesos industriales, de manera que este calor residual se pueda utilizar para alimentar un ciclo de turbina de vapor. En un sentido amplio, una planta de ciclo combinado consiste en la combinación de dos o más dispositivos trabajando en ciclos, que permitan una conversión más completa y eficiente de la energía aportada. Esto implica, por ejemplo, la unión de un ciclo de gas a alta temperatura con un ciclo de moderada temperatura, de manera que el calor residual del primero siva como calor de aporte para el segundo, como el dispositivo estudiado en Blanco y Peña (2011).

Un ciclo interesante, del hasta ahora no se conoce asociación a máquina real alguna, es el ciclo Zilch (Arrot, 1977; Zemansky y Ditman, 1986), estudiado con el objeto de mostrar la validez de la primera y segunda leyes de la termodinámica, analizando la variación de la entropía del sistema, en el marco teórico de la termodinámica clásica de equilibrio. Dicho ciclo, está formado por dos procesos isotérmicos conectados por una adiabática y una isocórica, con la condición de que la isocórica que conecta los procesos isotérmicos sea 
tal que el trabajo total del ciclo resulte cero. El ciclo Zilch se ha estudiado recientemente usando una aproximación para el proceso adiabático, tomando en cuenta que este proceso puede ser aproximado por una sucesión de procesos isobáricos seguidos de procesos isocóricos, de manera que el trabajo obtenido sea muy cercano al trabajo debido a la adiabática (Binder y Tanoue, 2013). Independientemente del valor del trabajo, se muestra la forma general del ciclo en la Figura 1, en el plano volumen vs presión $(V, p)$, y su respectiva transformación en el plano entropía vs temperatura $(S, T)$.
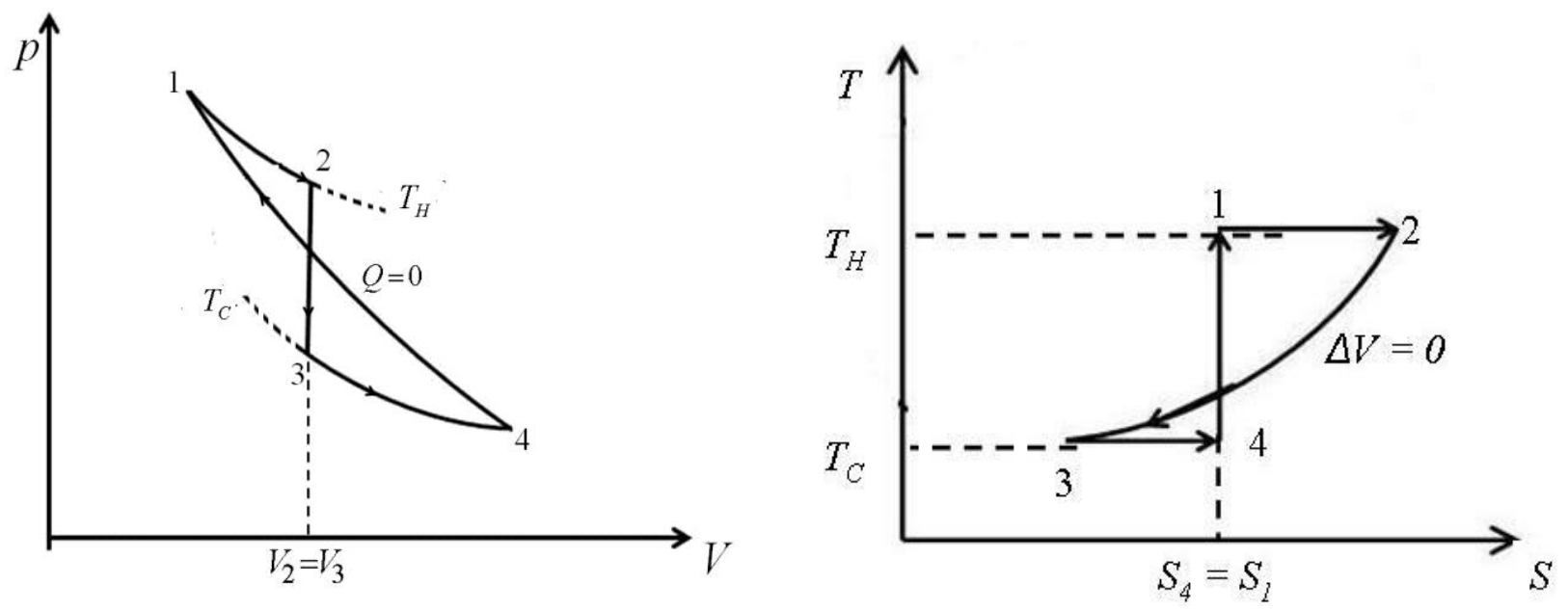

Fig. 1. Representación del ciclo zilch en el plano $(V, p)$ y en el plano $(S, T)$, respectivamente

En el presente trabajo se estudia el ciclo Zilch como la representación de un dispositivo que contenga un proceso de regeneración de calor, en el contexto de la termodinámica de tiempos finitos. Para realizar dicho estudio, consideraremos su necesaria modificación incluyendo explícitamente la diferencia entre temperaturas de trabajo y temperatura de la fuente calorífica. Se considera por comodidad un gas ideal como fluido de trabajo y, la transferencia de calor como la ley de enfriamiento de newton, puesto que la transferencia de calor por radiación no parece relevante cuando se transfiere energía vía quema de combustible, como es el caso de las centrales termoeléctricas y núcleo-eléctricas, y como se ha mostrado en diversos trabajos que estudian el calentamiento de la substancia de trabajo en diversos mecanismos, recientemente Izquierdo-Barrientos et al. (2016), Broatch et al. (2019) entre otros.

Las expresiones de potencia y eficiencia, obtenidas permiten considerar el ciclo como un modelo adecuado para un sistema termodinámico que pueda funcionar con regeneración de calor residual, produciendo suficiente calor para obtener una cantidad de trabajo extra, optimizando el uso de la energía suministrada al inicio del ciclo. Se hacen cambios de variable adecuados para estudiar el ciclo de Zilch modificado que se muestra a continuación. Las unidades de los parámetros involucrados han de considerarse en el sistema internacional de Unidades. Los resultados muestran la posibilidad de que el ciclo Zilch podría representar el desempeño de máquina que aproveche el calor residual (regeneración) para producir una segunda cantidad de trabajo, de manera semejante al dispositivo estudiado en Blanco y Peña (2011), pues se encuentran regiones de existencia física de la potencia y la eficiencia del ciclo, para las variables introducidas, de manera semejante a los ciclos conocidos de máquinas reales (Otto, Diesel, etc), que son idealizaciones de los ciclos reales de ellas.

\section{OTROS ANTECEDENTES}

Para realizar el estudio del ciclo Zilch, desde el punto de vista de la termodinámica de tiempos finitos, es conveniente tomar en cuenta que la función objetivo, la potencia del ciclo, se puede hacer siguiendo el modelo de Curzon y Ahlborn (1975) ó siguiendo el modelo de Gutkowicz-Krusin et al. (1978). La ventaja con este último es que las variables que se introducen son parámetros construidos con razones de temperaturas y por tanto sin unidades, y siempre han de estar dentro de un intervalo $(0,1)$. Y puesto que no es común en la literatura este procedimiento, a riesgo de pecar de excesivamente detallistas, mostraremos paso a paso dicho algoritmo aplicado al ciclo propuesto. Se utiliza además una simplificación de los parámetros variables como en Agrawal (2009) y Páez-Hernández et al. (2016). La modificación necesaria del ciclo Zilch de la Figura 1 se muestra en la Figura 2, en donde se hace explícita la diferencia entre las temperaturas de la fuente de calor y la debida al calor residual, $T_{H}$ y $T_{C}$, respectivamente, cumpliéndose que $T_{H}>T_{C} ;$ y las temperaturas 
de trabajo $T_{H W}$ y $T_{C W}$, siendo $T_{H W}>T_{C W}$. Como se sabe, el trabajo $W_{\text {tot }}$ total será la suma de los calores ganado y perdido por el sistema, $Q_{H}, Q_{C}$, respectivamente.

Se toma en cuenta que en el proceso isocórico el trabajo es nulo, y se toma en cuenta que el enfriamiento para dicho proceso puede suponerse en función de las temperaturas $T_{H W}$ y $T_{C W}$. Para las temperaturas $T_{C}$ y $T_{C W}$, consideradas muy cercanas, se supone que el calor residual en ese momento puede ser utilizado para producir el trabajo de 3 a 4 como se muestra en la Figura 2. La temperatura $T_{H}$ es la del único almacén externo de calor.

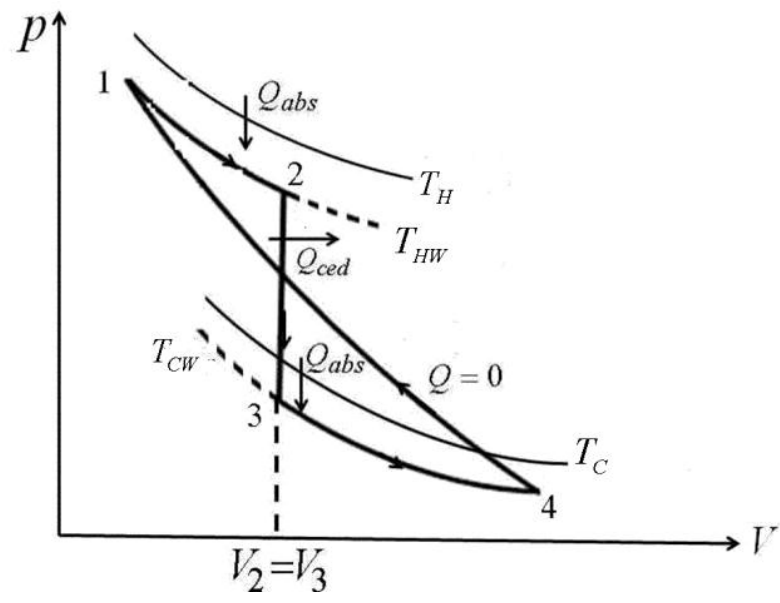

Fig. 2. Ciclo Zilch modificado en el plano $(V, p)$, como se requiere para su estudio a tiempo finito

Así, suponemos ahora un gas ideal como substancia de trabajo, de manera que para los procesos isotérmicos (en donde se transforma calor en trabajo) Utilizando una ley de transferencia de calor tipo ley de enfriamiento de Newton como en Izquierdo-Barrientos et al. (2016), Broatch et al. (2019), podemos escribir,

$$
W_{12}=Q_{H}=R T_{H W} \ln \left(V_{2} / V_{1}\right) \text { y } W_{34}=Q_{C}=R T_{C W} \ln \left(V_{4} / V_{3}\right)
$$

Como es evidente, $W_{\text {if }}$ representa el trabajo realizado durante el proceso del estado $i$ al estado $f$, particularmente el trabajo para el proceso isocórico es $W_{23}=0$. La constante $R$ es la de los gases ideales. Durante el proceso isocórico el sistema pierde la cantidad de calor, como se sabe,

$$
Q_{V}=C_{V}\left(T_{C W}-T_{H W}\right)
$$

en donde $C_{V}$ representa la capacidad calorífica a volumen constante. Así, tomando en cuenta que en la compresión adiabática el trabajo es $W_{41}=-C_{V}\left(T_{H W}-T_{C W}\right)$, se obtiene el trabajo total.

$$
W_{\text {tot }}=R T_{H W} \ln \left(V_{2} / V_{1}\right)+R T_{C W} \ln \left(V_{4} / V_{3}\right)-C_{V}\left(T_{H W}-T_{C W}\right)
$$

El calor total transformado en trabajo por el sistema durante las expansiones isotérmicas es $Q_{a}=Q_{H}+Q_{C}$. La eficiencia se puede escribir entonces a partir de su definición como,

$$
\eta=\frac{W_{t o t}}{Q_{a}}=\frac{R T_{H W} \ln \left(V_{2} / V_{1}\right)+R T_{C W} \ln \left(V_{4} / V_{3}\right)-C_{V}\left(T_{H W}-T_{C W}\right)}{R T_{H W} \ln \left(V_{2} / V_{1}\right)+R T_{C W} \ln \left(V_{4} / V_{3}\right)}
$$

Por otro lado, de acuerdo con la ley de enfriamiento de Newton, en general

$$
\frac{d Q}{d t}=\alpha\left(T_{i}-T_{f}\right) \Rightarrow Q=\alpha\left(T_{i}-T_{f}\right) t
$$

donde las temperaturas inicial y final del intercambio de calor son $T_{i}$ y $T_{f}$, respectivamente, con $T_{i}>T_{f}$. El tiempo de duración de dicho proceso entre cualquier sistema y sus fuentes de calor es $t, y \alpha$ es la conductancia térmica, que consideraremos igual en los dos procesos de expansión isotérmica. Esto es, los tiempos durante los procesos isotérmicos para el ciclo considerado son, 


$$
t_{1}=\frac{R T_{H W} \ln \left(V_{2} / V_{1}\right)}{\alpha\left(T_{1}-T_{1 w}\right)}, \text { y } t_{2}=\frac{R T_{C W} \ln \left(V_{4} / V_{3}\right)}{\alpha\left(T_{3}-T_{3 w}\right)}
$$

Para el proceso a volumen constante, utilizando la hipótesis de equilibrio local (De Groot y Mazur, 1962),

$$
\frac{d U}{d t}=\frac{d Q}{d t}=C_{V} \frac{d T}{d t}=r_{V} C_{V}
$$

y por tanto, siendo $r_{V}$ un parámetro medible,

$$
t_{V}=\frac{1}{r_{V}}\left(T_{C W}-T_{H W}\right)
$$

Como se observa en la Figura 2, el enfriamiento debe ser tal que se conserve suficiente energía calorífica para que pueda realizarse trabajo de expansión isotérmico. Asumiendo $K_{V}=1 / r_{v}$, como una tasa de enfriamiento promedio (Páez-Hernández y Angulo-Brown, 1996), y que el proceso adiabático es prácticamente instantáneo, se tiene que el tiempo total del ciclo es como,

$$
\tau=t_{1}+t_{2}+t_{V}=\frac{R T_{H W} \ln \left(V_{2} / V_{1}\right)}{\alpha\left(T_{H}-T_{H W}\right)}+\frac{R T_{C W} \ln \left(V_{4} / V_{3}\right)}{\alpha\left(T_{C}-T_{C W}\right)}-K_{V}\left(T_{C W}-T_{H W}\right)
$$

Al considerar el valor absoluto de la integración de (7), puesto que el tiempo debe ser positivo. De donde, ya que $V_{2}=V_{3}$ (ver Figura 2), y la potencia del ciclo se define como $P=W / t$, se tiene,

$$
P=\frac{R T_{H W} \ln \left(V_{3} / V_{1}\right)+R T_{C W} \ln \left(V_{4} / V_{3}\right)-C_{V}\left(T_{H W}-T_{C W}\right)}{\frac{R T_{H W} \ln \left(V_{3} / V_{1}\right)}{\alpha\left(T_{H}-T_{H W}\right)}+\frac{R T_{C W} \ln \left(V_{4} / V_{3}\right)}{\alpha\left(T_{C}-T_{C W}\right)}-K_{V}\left(T_{C W}-T_{H W}\right)}
$$

La expresión (10) puede simplificarse utilizando los cambios $u=T_{H W} / T_{H}, z=T_{C W} / T_{H W}$, y $\varepsilon=T_{C} / T_{H}$, en donde $u$ y $z$ son propiamente variables, $\varepsilon$ es la razón de las temperaturas conocidas extremas, y usando las propiedades de logaritmos se toma en cuenta que $\gamma=C_{p} / C_{V}$ es el exponente politrópico, y $C_{p}$ y $C_{V}$ son las capacidades caloríficas a presión y volumen constante, respectivamente. Así que,

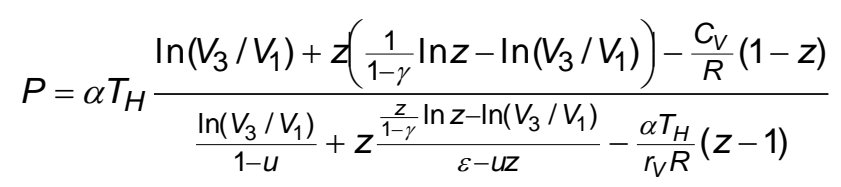

Definimos $r_{e}=V_{3} / V_{1}$ como una razón de expansión, y recordamos que las capacidades caloríficas, $C_{p}$ y $C_{V}$ se relacionan con la constante general de los gases como $C_{p}-C_{V}=R$, de manera que $1 /(\gamma-1)=C V / R$. Para obtener una expresión aproximada de (11) usamos la aproximación a primer orden de lnz, pues cumple con la condición $|z|<1$, de manera que se tiene

$$
\ln z=\ln [z+1-1]=\ln [1+(z-1)] \approx z-1,
$$

y por tanto (11) puede escribirse como,

$$
P \approx \alpha T_{H} \frac{(1-z)\left(\frac{C_{V}}{R}(z-1)+\ln r_{e}\right)(\varepsilon-z u)(1-u) R}{(\varepsilon-z u) R \ln r_{e}-z\left[\ln r_{e}+\frac{C_{V}}{R}(z-1)\right](1-u) R+K_{V} \alpha T_{H}(\varepsilon-z u)(1-u)(1-z)}
$$

Utilizando los mismos cambios en los parámetros involucrados, la eficiencia, Ec. (5), se escribe como,

$$
\eta=1-\frac{\frac{C_{V}}{R}}{\ln r_{e}+\frac{C_{V}}{R} z}=1-\frac{1}{(\gamma-1) \ln r_{C}+z}
$$


La Ecuación (13) muestra que la potencia del ciclo tiene valores físicamente posibles en la región $(0,1) \times(0,1)$, para las variables $u$ y $z$, como debe de ser, debido a su definición. También muestra las restricciones en los valores de estos parámetros variables, esto es su región de existencia física real. También la expresión obtenida para la eficiencia, En (14), se observa que el intervalo de existencia física de la eficiencia es $(0,1)$, como debe ser, pero su intervalo de existencia física real puede ser un sub-intervalo de $(0,1)$, pues depende del tipo de substancia de trabajo utilizada, debido a la aparición de un factor que incluye al exponente politrópico.

\section{RESULTADOS Y DISCUSIÓN}

Para hallar las regiones previamente mencionadas partimos de (13), en donde se observa que para la condición $P=0$ se obtienen las relaciones,

$$
\begin{aligned}
& \frac{C_{V}}{R} \ln r_{e}+(z-1)=0 \Rightarrow z=\ln r_{e}^{-R / C_{V}}+1 \\
& z=\varepsilon / u
\end{aligned}
$$

De la primera expresión en (15), encontramos que $z$ debe estar en un intervalo $\left(\left(-R / C_{V}\right) \ln r_{e}+1,1\right)$ y la región físicamente posible para la existencia de $P$ depende de la razón de expansión y del tipo de gas que se tenga como substancia de trabajo. Recordemos particularmente que en general para gases monoatómicos $\mathrm{Cp} / \mathrm{Cv}$ $=(3 / 2) R$ y para gases di-atómicos $C p / C v=(5 / 2) R$. Así que para las condiciones extremas $z=0$ y $z=1$ será posible hallar los valores extremos de la razón de expansión, y no puede ser arbitraria. Se encuentra,

$$
\begin{aligned}
& z=0 \Rightarrow r_{e}=\exp \left(\frac{C_{V}}{R}\right) \\
& z=1 \Rightarrow r_{e}=1
\end{aligned}
$$

De la segunda en (15), tomando en cuenta (16) encontramos que,

$$
u=\varepsilon / \exp \left(\frac{C_{V}}{R}\right) \text { ó } u=\varepsilon,
$$

Los parámetros variables en (13) son $z$ y $u$, los demás son parámetros medibles. Así, para efectos de comparación suponemos $\alpha=1$ (en $\mathrm{KJ} / \mathrm{K} \cdot \mathrm{t}$ ), considerando un valor adecuado para el cociente $r_{\mathrm{e}}=V_{3} / V_{1}$ (razón de expansión) y para $K_{V}=1 / r_{v}$ como los de un ciclo estándar de aire (Páez-Hernández y Angulo-Brown 1996), de manera que se obtiene la correspondiente gráfica para la potencia y la eficiencia. Para un gas monoatómico $C_{V}=(3 / 2) R, R=8.31 \mathrm{KJ} / \mathrm{Kg} \cdot \mathrm{K}$ (constante general de los gases) y $K_{V}=18.67 \times 10^{-6} \mathrm{sK}^{-1}$, se obtiene el comportamiento de la potencia y de la eficiencia, como se muestra en la Figura 3, respectivamente. En el caso de la potencia de salida, puesto que solo se quiere observar su comportamiento y no sus valore numéricos, se considera el graficar la expresión $P / \alpha T_{H}$.
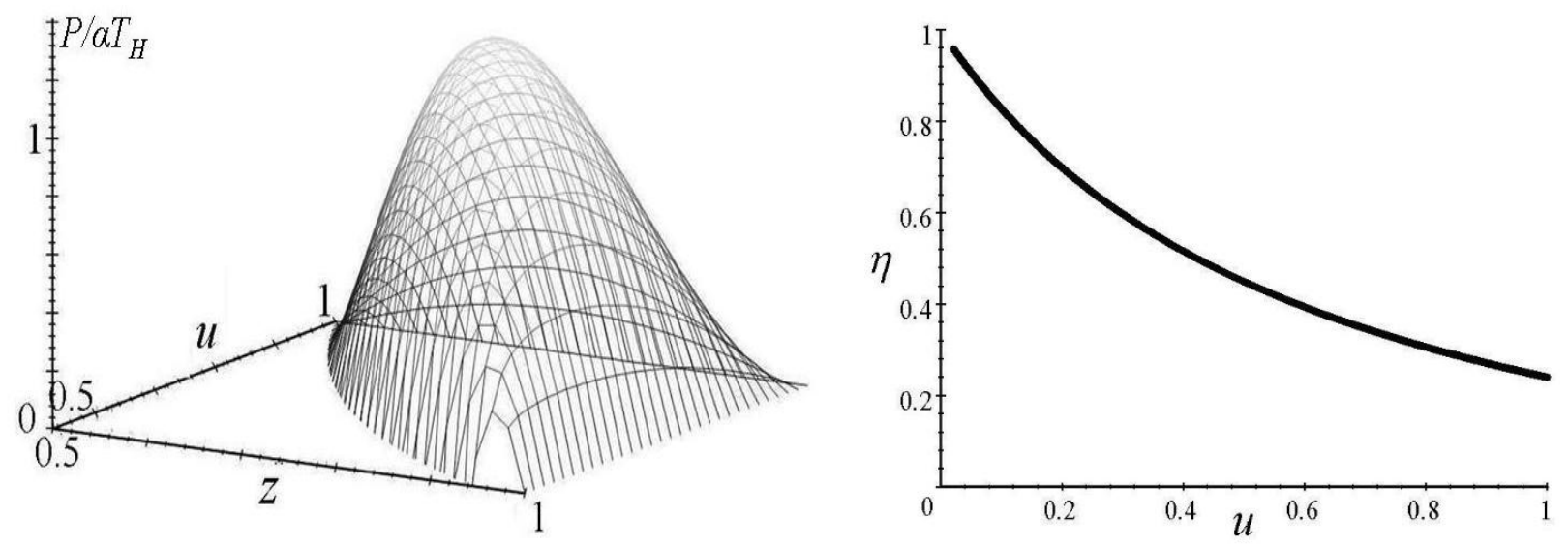

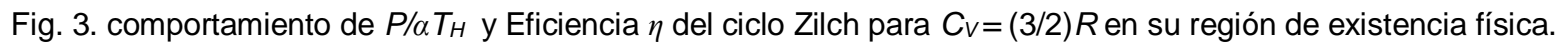

Para un gas di-atómico, donde $C_{V}=(5 / 2) R$, el comportamiento de la potencia es esencialmente el mismo que para $C_{V}=(3 / 2) R$, pero tanto el valor de $P$ como su región de existencia física son diferentes. Asimismo, la eficiencia tiene esencialmente el mismo comportamiento, pero sus valores en el intervalo de existencia física varían, como lo muestra la Figura 4, respectivamente. Es conveniente hacer notar que el análisis realizado muestra que la eficiencia y la potencia, como funciones de las variables $u$ y $z$, permiten describir el desempeño 
del ciclo, de manera análoga al estudio del ciclo de Curzon y Ahlborn en Gutkowicz-Krusin et al. (1978) y Ladino-Luna (2010), lo que no debe entenderse como una comparación, puesto que solo se han estudiado ambos ciclos con un mismo algoritmo.
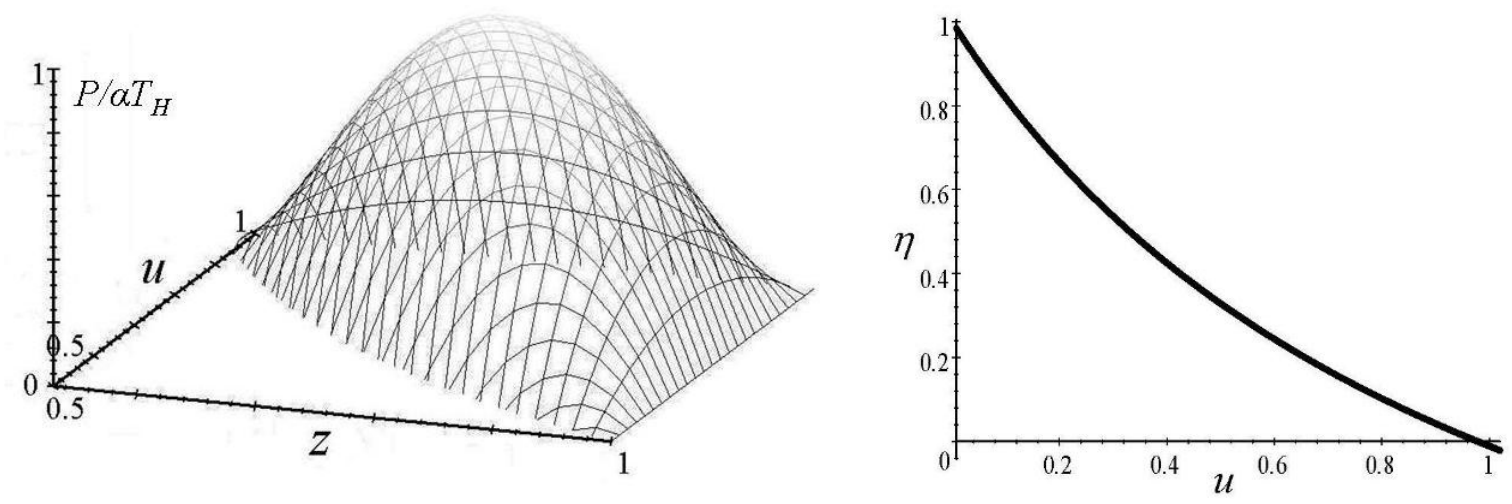

Fig 4. Comportamiento de $P / \alpha T_{H}$ y Eficiencia $\eta$ del ciclo Zilch para $C_{V}=(5 / 2) R$ en su región de existencia física.

Además, el interés de hacer este análisis ha sido mostrar una manera de modelar el funcionamiento de sistemas termodinámicos convertidores de calor en trabajo, que contengan un proceso regenerativo. Desafortunadamente no existen muchos trabajos que tomen como objetivo el estudio de este ciclo teórico (de Zilch), lo cual no impide que se puedan realizar otros estudios para el mismo, semejantes a diversas y recientes publicaciones, en el contexto de la termodinámica de tiempos finitos, como los de Feidt y Costea (2019), Oh et al. (2020), Chen et al. (2020), entre otros.

\section{CONCLUSIONES}

En el presente trabajo, a partir de los resultados obtenidos para el del ciclo Zilch modificado, incluyendo las fuentes de calor necesarias para representar adecuadamente la diferencia entre temperaturas de trabajo y temperaturas de dichas fuentes, se obtienen las siguientes conclusiones básicas:

Es posible describir el comportamiento de una máquina o planta de poder a través de la descripción del comportamiento de la potencia y la eficiencia, en términos de dos variables neutras (sin unidades de medida) y varios parámetros constantes medibles. Las condiciones de existencia (regiones de existencia física) de estas cantidades permiten estudiar el desempeño de tales sistemas termodinámicos.

El comportamiento general de la potencia y la eficiencia mostrados confirman la posibilidad de que el ciclo Zilch pueda representar un mecanismo que aproveche el calor residual, permitiendo una producción extra de trabajo.

La termodinámica de tiempos finitos, como paradigma para estudios de sistemas termodinámicos, ha permitido el desarrollo de nuevas metodologías y nuevos algoritmos, entre los que se encuentran los utilizados en el presente trabajo y en la literatura citada, así como el estudio de diversos ciclos susceptibles de ser vir de modelo a sistemas termodinámicos reales, como el ciclo aquí estudiado.

\section{AGRADECIMIENTOS}

DLL y PHRT agradecen el apoyo total de la Universidad Autónoma Metropolitana_A, JCCE agradece a CONACYT, EDI-IPN y COFAA-IPN.

\section{REFERENCIAS}

Agrawal, D.C., A Simplified Version of the Curzon-Ahlborn Engine, https://doi:10.1088/0143-0807/30/5/024, Eur. J. Phys., 30, 1173-1179 (2009)

Angulo-Brown, F., An Ecological Optimization Criterion for Finite Time Heat Engines, https://doi.org/10.1063/1.347562, J. Appl. Phys., 69, 7465-7469 (1991)

Angulo-Brown, F., Fernandez-Betanzos, J., y Díaz-Pico C.A., Compression Ratio of an Optimized Air Standard Otto-Cycle Model, https://doi:10.1088/0143-0807/15/1/007, Eur. J. Phys., 15, 38-42 (1994)

Arrot, A.S., The Zilch Cycle: An Application of the First Law of Thermodynamics, https://doi.org/10.1119/1.10809, Am. J. Phys., 45(7), 672-673 (1977) 
Binder, P.M., y Tanoue, C.K.S., Variations on the Zilch Cycle, https://doi.org/10.1119/1.4820862, Phys. Teach., 51, 434$436(2013)$

Blanco, J.M., y Peña, F., Incremento de la Eficiencia en Centrales Termoeléctricas por Aprovechamiento de los Gases de la Combustión, http://dx.doi.org/10.4067/S0718-07642011000400003, Inf. Tecnol., 22(4), 15-22 (2011)

Broatch, A., Olmeda, P., y otros dos autores, New Approach to Study the Heat Transfer in Internal Combustion Engines by 3D Modeling, https://doi.org/10.1016/j.ijthermalsci.2019.01.006, Int. J. Therm. Sci., 138, 405-415 (2019)

Curzon, F.L., y Ahlborn, B., Efficiency of a Carnot Engine at Maximum Power Output, https://doi.org/10.1119/1.10023, Am. J. Phys., 43, 22-24 (1975)

Chambadal, P., Récupération de chaleur 'a la Sortie d’un Reactor, Cap. 3, 39-58. Armand Colin (Ed.), Francia (1957)

Chen, L., Ge, Y., y otros tres autores, Performance of Universal Reciprocating Heat-Engine Cycle with Variable Specific Heats Ratio of Working Fluid, https://doi.org/10.3390/e22040397, Entropy, 22, 397_1-13 (2020)

De Groot, S.R. y Mazur, P., Non-Equilibrium Thermodynamics, Cap. 3, 20-28. North-Holland, Holanda (1962)

Feidt, M. y Costea, M., Progress in Carnot and Chambadal Modeling of Thermomechanical Engine by Considering Entropy Production and Heat Transfer Entropy, https://doi.org/10.3390/e21121232, Entropy, 21, 1232_1-13 (2019)

Gutkowicz-Krusin, D., Procaccia, I., y Ross, J., On the Efficiency of Rate Processes. Power and Efficiency of Heat Engines, https://doi.org/10.1063/1.437127, J. Chem. Phys., 69, 3898-3906 (1978)

Izquierdo-Barrientos, M.A., Sobrino, C., y Almendros-Ibáñez, J.A., Modeling the Heat Transfer Coefficient between a Surface and Fixed and Fluidized Feds with Phase Change Material, Journal of heat transfer (Transactions of the ASME), 138(7): 072001, 11 pages, https://doi.org/10.1115/1.4032981, (2016)

Ladino-Luna, D., Análisis del Comportamiento de un Ciclo Tipo Carnot, http://dx.doi.org/10.4067/S071807642010000400011, Inf. Tecnol., 21(4), 79-86 (2010)

Ladino-Luna, D., Portillo-Díaz, P., y Páez-Hernández, R.T., Local Stability of a Curzon-Ahlborn Cycle with a Non-Linear Heat Transfer Law for Maximum Power Output Regime, http://dx.doi.org/10.4236/jmp.2013.47A2004, J. Modern Physics, 4, 22-27 (2013)

Lanzetta, M., Rossi, A., y Puppato, A., Modelling Activity Times by Hybrid Synthetic Method, http://dx.doi.org/10.1080/09537287.2016.1159351, Production Planning \& Control, 27(11), 909-924 (2016)

Lefft. H.S., Thermal Efficiency at Maximum Work Output: New Results for Old Heat Engines., https://doi.org/10.1119/1.15071, Am. J. Phys., 55(7), 602-610 (1987)

Merchán, R.P., Santos, M.J., y otros cuatro autores, A., On-Design Pre-Optimization and Off-Design Analysis of Hybrid Brayton Thermosolar Tower Power Plants for Different Fluids and Plant Configurations, https://doi.org/10.1016/j.rser.2019.109590, Renewable and Sustainable Energy Reviews, 119 (2020)

Mozurkewich, M., y Berry, S., Optimal Paths for Thermodynamic Systems: The Ideal Otto Cycle, https://doi.org/10.1063/1.329894, J. Appl. Phys., 53, 34-42 (1982)

Novikov, I.I., The Efficiency of Atomic Power Stations, https://doi.org/10.1016/0891-3919(58)90244-4, J. Nuclear Energy II, Vol. 7, 125-128 (1958)

Oh, S., Baggag, A., y Nha, H., Entropy, Free Energy, and Work of Restricted Boltzmann Machines, https://doi.org/10.3390/e22050538, Entropy, 22, 538_1-15 (2020)

Páez-Hernández, R.T. y Angulo-Brown, F., Eficiencia y Relación de Compresión en un Ciclo Diesel a Tiempo Finito, Rev. Mex. Fís., 42, 684-691 (1996)

Páez-Hernández, R.T., Chimal-Eguía J.C., y otros dos autores, Comparative Performance Analysis of a Simplified CurzonAhlborn Engine, https://doi.org/10.3390/e20090637, Entropy, 20, 637_1-9 (2018)

Páez-Hernández, R.T., Portillo-Díaz, P., y otros tres autores, An Analytical Study of the Endoreversible Curzon-Ahlborn Cycle for a Non-Linear Heat Transfer Law, https://doi.org/10.1515/jnet-2015-0032, J. Non-Equilib. Thermodyn., 41(1), 1927 (2016)

Ponmurugan, M., Realistic Thermal Heat Engine Model and its Generalized Efficiency, arXiv:1912.12949v1, [condmat.stat-mech], 1-5 (2019)

Rubin, M., Optimal Configuration of an Irreversible Heat Engine with Fixed Compression Ratio, https://doi.org/10.1103/PhysRevA.22.1741, Phys. Rev. A, 22, 1741-1752 (1980)

Santillán, M., Maya, G., y Angulo-Brown, F., Local Stability Analysis of an Endorreversible Curzon-Ahlborn-Novikov Engine Working in a Maximum-Power-Like Regime, https://doi.org/10.1088/0022-3727/34/13/318, J. Phys D: Appl. Phys., 34, 2068-2074 (2001)

Zemansky M.W. y Ditman, R.H., Heat and Thermodynamics. Versión en castellano: Calor y Termodinámica, 225-226. McGraw-Hill, México (1985)

Zhu F., Chen, L., y Wang, W., Thermodynamic Analysis and Optimization of an Irreversible Maisotsenko-Diesel Cycle, https://doi.org/10.1007/s11630-019-1153-1, J. Therm. Sci., 28(4), 659-658 (2019) 